\title{
Effects of the 2010 coral bleaching on phylogenetic clades and diversity of zooxanthellae (Symbiodinium spp.) in soft corals of the genus Sinularia
}

\author{
ThadSIn PANITHANARAK* \\ Institute of Marine Science, Burapha University, Saensook, Muang, Chonburi 20131, Thailand
}

Received 4 July 2013; Accepted 23 October 2014

\begin{abstract}
The mass coral bleaching event in South East Asian countries in 2010 caused severe damage to reef dwellers on the coast of the Andaman Sea and the Gulf of Thailand. Bleaching is a process where the symbiosis between corals and zooxanthellae (dinoflagellates of the genus Symbiodinium) breaks down. The symbiosis between Symbiodinium and corals is essential to the productivity, survival and success of corals. Symbiodinium associated with coral hosts is classified into six genetically distinct clades based on nuclear ribosomal DNA and partial chloroplast large subunit rDNA. Each clade possesses unique environmental, ecological and geographic variations, influencing the resilience of corals to elevated temperatures and bleaching. This study was the first field observation of changes in Symbiodinium communities in the Gulf of Thailand over two years including a natural bleaching event from late April to June, 2010. Phylogenetic clades and diversity of Symbiodinium inhabiting soft corals of the genus Sinularia were investigated using the analysis of chloroplast gene sequence variation at the ribosomal large subunit 23S Domain V. A total of 69 Sinularia spp. samples were obtained from two sampling sites off Jorake and Juang Islands (Sattahip, Chonburi Province) during two sampling periods: before (November 2008-January 2010, $n=45$ ) and after (November 2010, $n=24$ ) the 2010 coral bleaching event. The results revealed a significant change in the Symbiodinium community after the bleaching event. Before bleaching, most of Symbiodinium clade D was detected from Jorake Island while clade $\mathrm{C}$ was dominant in the Juang Islands. In contrast, after bleaching, only clade D was identified from both sampling sites.
\end{abstract}

Key words: chloroplast gene, coral bleaching, Sinularia, Symbiodinium, the Gulf of Thailand

\section{Introduction}

During late April to June 2010, mass coral bleaching events in South East Asian countries caused severe damage to reef dwellers along the coasts of the Andaman Sea and the Gulf of Thailand as sea surface temperatures anomalies in the range of $30-34^{\circ} \mathrm{C}$ were recorded. Several reef surveys conducted by the Department of Marine and Coastal Resources in collaboration with a network of Thai universities and NGOs revealed widespread (over 80\%) and severe damage on most reef sites (Yeemin et al. 2012). In the Gulf of Thailand, coral bleaching was observed in Kood Island, Chang Island, Man Islands, Samae-San Is-

\footnotetext{
*Corresponding author: Thadsin Panithanarak; E-mail, thadsin@hotmail. com

This is a report of research presented at the 1st Asian Marine Biology Symposium (AMBS) held in 2012 in Phuket, Thailand, co-edited with the cooperation of guest editors Drs M. Nakaoka and M. Shimanaga.
}

lands and nearby, but was less severe and less extensive compared with the Andaman Sea (Yeemin et al. 2010). Not only hard corals suffered from the bleaching event, other marine invertebrates forming mutualistic symbioses with zooxanthellae (e.g. soft corals, zoanthids and giant clams) were also bleached (Tun et al. 2010, Yeemin 2010).

The symbiosis between Symbiodinium (a genus of unicellular dinoflagellates also referred to as zooxanthellae) and corals is essential to the productivity, survival and success of the coral hosts (Muscatine \& Porter 1977). Bleaching is a process whereby this symbiosis breaks down. Advanced molecular studies reveal that the genus Symbiodinium is highly diverse and is classified into 9 genetically distinct clades (clades A to I; Pochon \& Gates 2010) based on nuclear ribosomal DNA (rDNA) and partial chloroplast large subunit rDNA sequence variations. Among these, six clades (clades A to D, F and G) were reported to form mutualistic symbiosis with coral hosts (LaJeunesse et al. 2010). Each clade possesses unique environmental, ecolog- 
ical and geographic variations, influencing the resilience of coral hosts to elevated temperatures and bleaching. Few studies (e.g. LaJeunesse et al. 2010, Panithanarak 2012) have described compositions of Symbiodinium communities in coral hosts from the Andaman Sea and the Gulf of Thailand. Symbiodinium clades C and D were reported from the Andaman Sea (Cape Panwa, Koh Hae, Koh Phi Phi Don, Koh Phi Phi Lae, Koh Racha and the Similand Islands) and the Gulf of Thailand (Nang-Rong Beach, Jorake, Juang and Jan Islands) with the thermally resistant clade D being the most common type in the Andaman Sea, especially at inshore reef sites (LaJeunesse et al. 2010). Similar to the Andaman Sea, clade D was abundant at inshore reef sites (Nang-Rong Beach and Jorake Island) in the Gulf of Thailand (Panithanarak 2012). Clade D Symbiodinium was more diverse and occurred frequently in coral hosts from inshore locations compared with clade $\mathrm{C}$, most likely as a result of high temperature and turbid conditions of the inshore relative to offshore locations (LaJeunesse et al. 2010, Panithanarak 2012). However, none of the studies so far has examined the impact of high temperature or coral bleaching on changes in clades of Symbiodinium communities in Thailand. As coral bleaching is predicted to increase in frequency and severity owing to climate change (Burke et al. 2011, Hoegh-Guldberg 2011), understanding and monitoring the response of Symbiodinium to elevated temperature and bleaching is required for developing strategies and to minimize the effects of climate change on coral reef ecosystems.
The present study is the first report monitoring changes in the Symbiodinium community within the Gulf of Thailand covering a two-year period, which included the mass coral bleaching event during late April to June, 2010. Phylogenetic clades and diversity of Symbiodinium (genus $\mathrm{Si}$ nularia, Family Alcyoniidae) inhabiting an abundant soft coral in the Gulf of Thailand were examined from two reef sites of Jorake and Juang Islands (coral reefs affected by bleaching in the nearby Samae-San Islands, Sattahip, Chonburi Province) by analyzing partial chloroplast large subunit rDNA sequence variations. Symbiodinium communities from two sampling periods, pre and post-bleaching, were monitored and compared. The aims are to investigate any changes in Symbiodinium community assemblages as a consequence of the bleaching event, and to identify Symbiodinium clades resistant to coral bleaching.

\section{Materials and Methods}

\section{Soft coral sampling}

Soft coral colonies of Sinularia spp. were collected randomly by scuba diving from two sampling sites off Jorake and Juang Islands (Sattahip, Chonburi Province, the Gulf of Thailand; Fig. 1) during two sampling periods: before the coral bleaching (November 2008 to January 2010, $\mathrm{n}=45$ ) and seven months after the coral bleaching (November 2010, $\mathrm{n}=24)$. Pieces of colony lobes $(0.5-1.0 \mathrm{~cm}$, $2-3$ pieces/colony) were removed and preserved in absolute

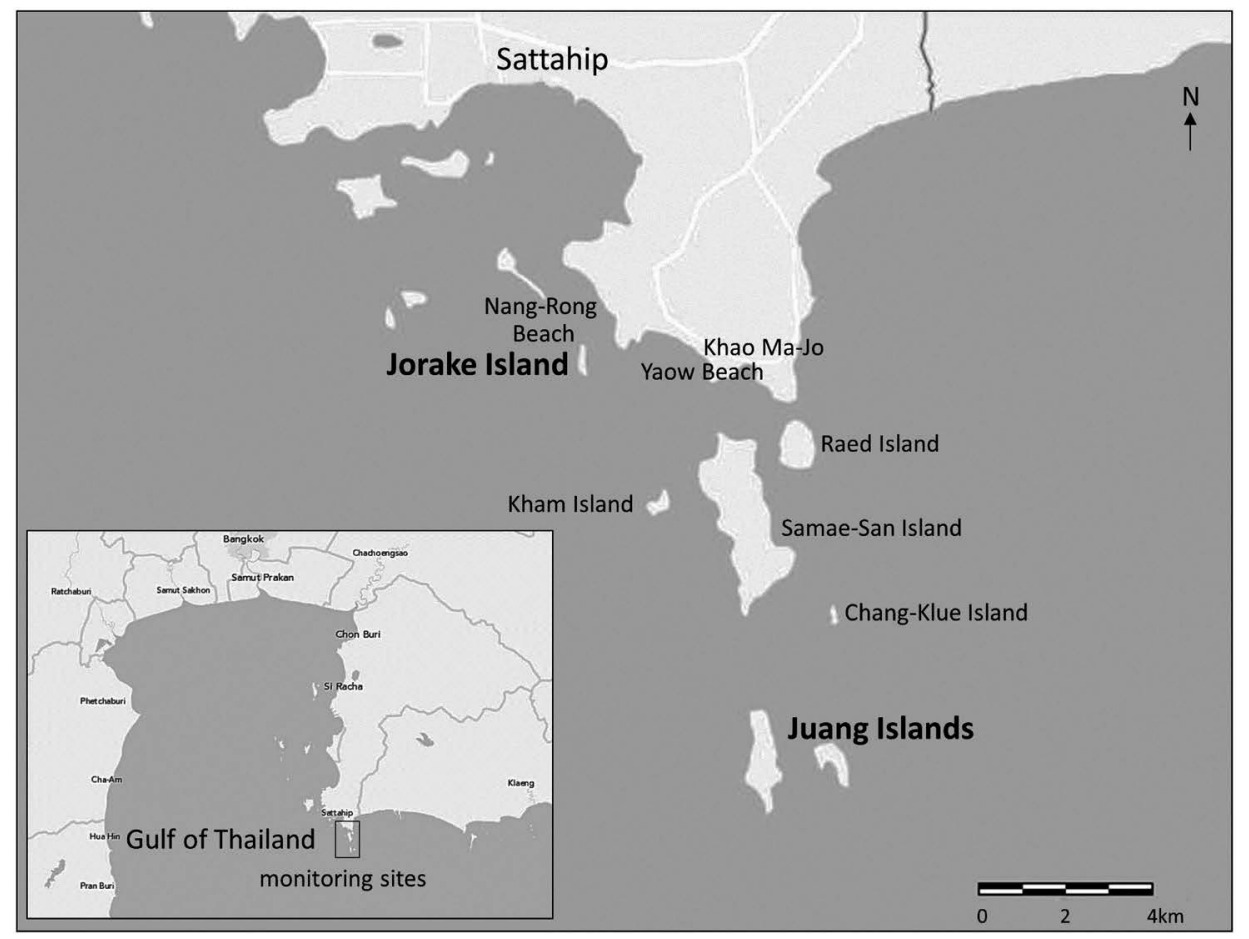

Fig. 1. Map showing Symbiodinium monitoring sites of Jorake and Juang Islands (Sattahip, Chonburi Province) modified from a map of Amphur Sattahip by Esri Map. 
ethanol for molecular analysis. Pre-bleaching specimens were obtained from the study by Panithanarak et al. (2011).

\section{DNA extraction, amplification and sequencing}

DNA was extracted from ethanol-preserved tissues using QIAamp DNA Minikits (Qiagen, Hilden, Germany). PCR amplification of chloroplast ribosomal large subunit 23S Domain V (cp23S rDNA) used the specific Symbiodinium primers $\mathrm{cp} 23 \mathrm{~s} 1$ (forward primer): 5'-GCTGTAACTATAACGGTCC-3' and cp23s2 (reverse primer): 5'-CCATCGTATTGAACCCAGC-3' (Oliver \& Palumbi 2011). The PCR protocols were modified from Zhang et al. (2000) with a $50 \mu \mathrm{L}$ reaction volume containing $0.2 \mathrm{mM}$ dNTP (Promega, Madison, WI, USA), 1xPCR Buffer, 50 nmol primer (Macrogen Inc., Seoul, Korea), $2.0 \mathrm{mM}$ $\mathrm{MgCl}_{2}, 1.5$ unit platinum Taq DNA polymerase (Invitrogen, Carlsbad, CA, USA) and $0.1-1.0 \mathrm{ng} \mu^{-1} \mathrm{~L}^{-1}$ template DNA. The PCR products were further purified using QIAquick PCR purification kits (Qiagen). Direct sequencing of purified PCR products was carried out by the sequencing service, Macrogen (Seoul, Korea) using both primers (cp23s1 and cp23s2).

\section{Data analysis}

Nucleotide sequences of cp23S rDNA were manually checked for accuracy. In each sample, two sequences (forward and reverse) were obtained and assembled using CAP3 (Huang \& Madan 1999; http://pbil.univ-lyon1.fr/ cap3.php). All sequences were compared with existing databases of Symbiodinium cp23S rDNA deposited in GenBank using BLASTN (http://blast.ncbi.nlm.nih.gov) and aligned using Clustal X 1.81.msw (Thompson et al. 1997). They were assigned to major clade identities $\mathrm{C}$ or $\mathrm{D}$ on the basis of showing highest identity with the respective database sequences. Haplotype frequencies of Symbiodinium clades C and D collected before and after the 2010 coral bleaching were calculated and compared using Arlequin 3.5 (Excoffier et al. 2005). Levels of genetic differentiation between pre and post-bleaching Symbiodinium communities was also estimated with $F_{\mathrm{ST}}$ (molecular distance: pairwise difference) using Arlequin 3.5.

\section{Phylogenetic analyses}

The sequence data used for phylogenetic analyses included all haplotypes identified from this study and 16 cp23S rDNA sequences of clade $\mathrm{C}$ and $\mathrm{D}$ symbionts from various coral hosts deposited in GenBank and/or as published (Santos et al. 2002, Pochon et al. 2006, Cantin et al. 2009, Stat et al. 2009, Pochon et al. 2010, Oliver \& Palumbi 2011, Pochon et al. 2012, Jeong et al. 2014). A neighborjoining (NJ) phylogenetic tree (Saitou \& Nei 1987) based on the cp23S rDNA gene was constructed using the distance method based on the Kimura two parameter substitution model (Kimura 1980). NJ trees were constructed using PAUP* 4.0 beta 10 (Swofford 2002) and rooted using the midpoint method. To assess the statistical significance of internal branching, a thousand bootstrap replicates were performed.

\section{Results}

As cp23S rDNA shows length variation across Symbiodinium clades A, C and D (Oliver \& Palumbi 2011), clades were first identified by visualization of band lengths on the agarose gels (compared with a standard 100 bp DNA ladder). In general, both clades $\mathrm{C}$ and $\mathrm{D}$ were detected from 45 Sinularia host samples collected before the 2010 coral bleaching event. Among 45 samples, three (6.67\%) showed multiple bands on a gel, indicating mixed symbiont clades $\mathrm{C}$ and $\mathrm{D}$ within a single sample. Clade $\mathrm{D}$ was unique for Jorake Island $(n=16, C=0, D=16)$ while clade $C$ was dominant in offshore reefs of the Juang Islands $(n=29, C=20$, $\mathrm{D}=6, \mathrm{C}+\mathrm{D}=3$, Fig. 2). Seven months after the bleaching event, only clade $\mathrm{D}$ was detected from both sampling sites off Jorake and Juang Islands (Fig. 2). When clade identities and frequencies were compared between pre and postbleaching Symbiodinium communities, a significant change in composition of Symbiodinium clades from Juang Islands after the bleaching event was obvious (see Fig. 2).

Following analysis of partial cp23S rDNA sequence variations (66 sequences analyzed excluding 3 mixed clade samples), Symbiodinium clades C and D were assigned on the basis of highest identity with the respective cp23S rDNA Symbiodinium sequences in Genbank. Symbiodinium clade identities were consistent with the previous clade identification by cp23S length variation. Among the 66 cp23S rDNA sequences, 661 nucleotides in length (prebleaching $n=42$, post-bleaching $n=24$ ), 4 haplotypes were identified with 3 and 1 haplotypes belonging to clade D and clade $\mathrm{C}$, respectively. The sequences of clade $\mathrm{D}$ and $\mathrm{C}$ haplotypes in this study were deposited in GenBank (accession numbers KJ801955-KJ801958). One clade C haplotype obviously differed from other haplotypes of clade D in 168 nucleotide substitutions mostly derived from insertions or deletions (indels). In contrast, nucleotide variation within clade D was low, with indels of only $1 \mathrm{bp}$. Before the bleaching event, clade C and D haplotypes were detected at comparable frequencies (relative frequencies: clade $\mathrm{C}$ haplotype $=0.452$; clade D haplotype $=0.500$ ), although after the bleaching event only clade D haplotypes were apparent. In addition, the Symbiodinium population of before the bleaching event was genetically different from the post-bleaching population $\left(F_{\mathrm{ST}}=0.37\right.$, $P<0.00001$; no. of permutations $=1,023$ ).

A neighbor-joining phylogenetic tree based on 4 haplotypes of symbiont clades $\mathrm{C}$ and $\mathrm{D}$ in this study and 16 cp23S rDNA sequences of symbiont clades $\mathrm{C}$ and $\mathrm{D}$ from various coral hosts deposited in GenBank is shown in Figure 3. Phylogenetic analysis revealed differentiation of 2 genetically distinct clades $\mathrm{C}$ and D. Each haplotype identified from this study was clustered within its own clade. The clade D cluster was strongly supported by bootstrap 



Fig. 2. Frequencies of Symbiodinium clades (C, D and a mixture of C and D) inhabiting a soft coral host, genus Sinularia from Jorake and Juang Islands (Sattahip, Chonburi Province). The frequencies are compared between the two monitoring periods: (a) before the 2010 coral bleaching event and (b) 7 months after the bleaching event. Size of pie charts is in proportion to the sample size.

values (see Fig. 3).

\section{Discussion}

The present study revealed a significant change in composition of Symbiodinium clades inhabiting Sinularia hosts from the Juang Islands after the 2010 coral bleaching event. Before the bleaching event, $68.97 \%$ of the randomly obtained samples harbored Symbiodinium clade C, while the remainder harbored clade $\mathrm{D}$ and a mixture of clades $\mathrm{C}$ and D (Fig. 2). After the bleaching event, surviving samples retained only heat-resistant clade D Symbiodinium. 


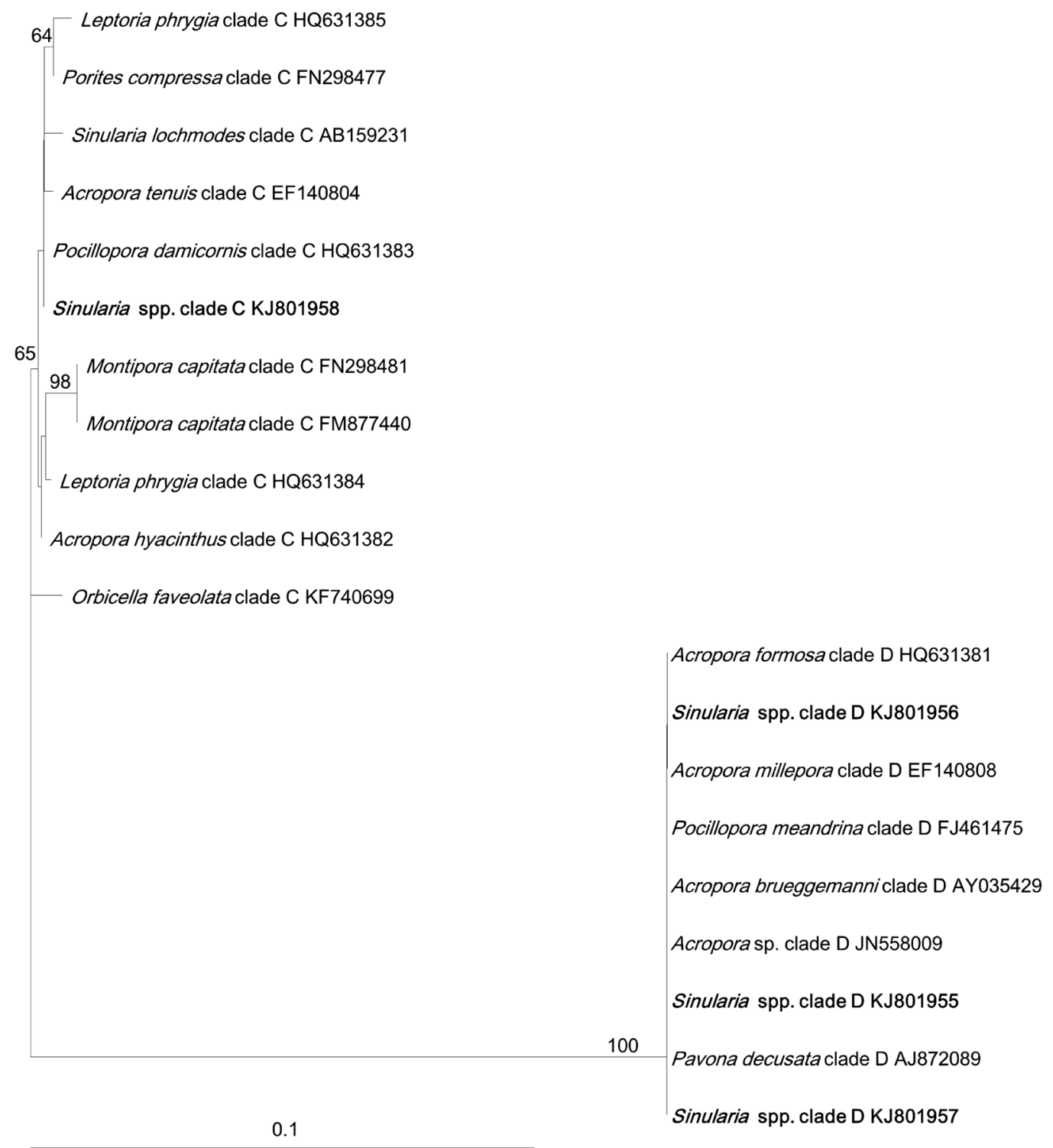

Fig. 3. A neighbor-joining clade credibility tree of the relationships of 4 Symbiodinium clade C and D haplotypes in this study and $16 \mathrm{cp} 23 \mathrm{~S}$ rDNA sequences of symbiont clades $\mathrm{C}$ and D from various coral hosts deposited in GenBank. The tree was rooted using the midpoint method. Numbers above internal branches are bootstrap values based on 1,000 replicates. Data from this study are in bold and provided with species names of the coral hosts (Sinularia spp.) followed by symbiont clades and GenBank accession numbers (KJ801955-KJ801958). For the reference data, species names of the coral hosts are followed by symbiont clades and GenBank accession numbers.

Loss of clade $\mathrm{C}$ or an increase of clade $\mathrm{D}$ after bleaching could possibly be explained by differential mortality of hosts harboring sensitive symbiont types (clade C) or symbiont changes within soft coral colonies (Symbiodinium shuffling or switching). Symbiont shuffling refers to changes in the relative abundance of each Symbiodinium clade/subclade under the influence of the environment. This change in symbiont community supports the Adaptive Bleaching Hypothesis $(\mathrm{ABH})$ of Buddemeier \& Fautin (1993) and Buddemeier et al. (2004), in which coral hosts switch or shuffle their thermally sensitive Symbiodinium for more tolerant clades/subclades during bleaching. How- ever, this change may come with coral fitness trade-offs such as lower skeletal growth (Jones \& Berkelmans 2010) and loss of photosynthetic efficiency (Rowan 2004).

Several other studies have also shown increases in the abundance of Symbiodinium clade D in coral hosts following bleaching events (Baker et al. 2004, Rowan 2004, Berkelmans \& van Oppen 2006, LaJeunesse et al. 2007, Jones et al. 2008). The field evidence of changes in Symbiodinium communities in tagged colonies of Acropora millepora before and after a natural bleaching event in 2006 in the Keppel Islands on the southern Great Barrier Reef (sGBR) suggested both symbiont shuffling and differential 
mortality as explanations for the changes in symbiont communities (Jones et al. 2008). However, another field observation in tagged colonies of Stylophora pistillata at Heron Island (sGBR) supported differential mortality of hosts harboring thermally sensitive symbionts rather than symbiont shuffling within a single host colony (Sampayo et al. 2008). In the present study, none of the colonies of Sinularia was tagged, so sampling before and after the 2010 coral bleaching event was random. As a consequence of this random sampling of host colonies, neither differential mortality nor symbiont shuffling explanations of the change in Symbiodinium communities after bleaching can be ruled out.

There was no change in Symbiodinium community from Jorake Island after the bleaching event as Sinularia hosts normally harbored only heat-tolerant Symbiodinium clade D at inshore locations (Panithanarak 2012). Jorake Island is close to Nang-Rong Beach (Fig. 1) while the Juang Islands are farther out at approximately 7-8 nautical miles (13-15 $\mathrm{km}$ ) from the coast. Therefore, stressful environments in Jorake Island (e.g. turbid sea water and large tidal exchanges) influence the abundance and evolutionary success of clade D over clade C. Several studies indicate that clade D Symbiodinium is most common in coral hosts from inshore locations with turbid environments (van Oppen et al. 2005, Mostafavi et al. 2007, LaJeunesse et al. 2010).

\section{Acknowledgements}

This study was funded by a budget for research promotion from the Thai Government to Burapha University in 2013. Part of the soft coral specimens used in this study were collected under the research project "Morphological and genetic variations of soft coral, Sinularia May, 1898 (Octocorallia: Alcyonacea) along Nang-Rong Beach, Jorake Island and the Juang Islands, Amphur Sattahip, Chonburi Province" (under the Plant Genetic Conservation Project under the Royal initiative of Her Royal Highness Princess Maha Chakri Sirindhorn) funded by a budget for research promotion from the Thai Government to Burapha University during 2009-2010.

\section{References}

Baker AC (2003) Flexibility and specificity of coral-algal symbiosis: diversity, ecology, and biogeography of Symbiodinium. Annu Rev Ecol Syst 34: 661-689.

Baker AC, Starger CJ, McClanahan TR, Glynn PW (2004) Corals adaptive response to climate change. Nature 430: 741.

Berkelmans R, van Oppen MJH (2006) The role of zooxanthellae in the thermal tolerance of corals: A "nugget of hope" for corals reefs in an era of climate change. Proc R Soc Lond Ser B 273: 2305-2312.

Buddemeier RW, Baker AC, Fautin DG, Jacobs JR (2004) The adaptive hypothesis of bleaching. In: Coral health and disease (eds Rosenberg E, Loya Y). Springer ch. 24, Berlin, Germany, pp. 427-444.

Buddemeier RW, Fautin DG (1993) Coral bleaching as an adaptive mechanism: a testable hypothesis. Bioscience 43: 320.

Burke L, Reytar K, Spalding M, Perry A (2011) Reefs at Risk revisited. World Resources Institute, Washington DC, 114 pp.

Cantin NE, van Oppen MJ, Willis BL, Mieog JC, Negri AP (2009) Juvenile corals can acquire more carbon from high-performance algal symbionts. Coral Reefs 28: 405-414.

Excoffier L, Laval G, Schneider S (2005) Arlequin ver. 3.0: An integrated software package for population genetics data analysis. Evol Bioinform Online 1: 47-50.

Hoegh-Guldberg O (2011) Coral reef ecosystems and anthropogenic climate change. Reg Environ Change 11: S215-S227.

Huang X, Madan A (1999) CAP3: A DNA sequence assembly program. Genome Res 9: 868-877.

Jeong HJ, Lee SY, Kang NS, Yoo YD, Lim AS, Lee MJ, Kim HS, Yih W, Yamashita H, LaJeunesse TC (2014) Genetics and morphology characterize the dinoflagellate Symbiodinium voratum, n. sp., (Dinophyceae) as the sole representative of Symbiodinium clade E. J Euk Microbiol 61: 75-94.

Jones AM, Berkelmans R (2010) Potential coasts of acclimatization to a warmer climates: Growth of a reef coral with heat tolerant vs. sensitive symbiont types. PLoS ONE 5: e10437. doi:10.1371/journal.pone.0010437.

Jones AM, Berkelmans R, van Oppen MJH, Mieog JC, Sinclair W (2008) A community change in the algal endosymbionts of a scleractinian coral following a natural bleaching event: field evidence of acclimatization. Proc R Soc B 275: 1359-1365.

Kimura M (1980) A simple method for estimating evolutionary rate of base substitutions through comparative studies of nucleotide sequences. J Mol Evol 16: 111-120.

LaJeunesse TC, Pettay DT, Sampayo EM, Phongsuwan N, Brown B, Obura DO, Hoegh-Guldberg O, Fitt WK (2010) Long-standing environmental conditions, geographic isolation and hostsymbiont specificity influence the relative ecological dominance and genetic diversification of coral endosymbionts in the genus Symbiodinium. J Biogeogr 37: 785-800.

LaJeunesse TC, Reyes-Bonilla H, Warner ME (2007) Spring bleaching among "Pocillopora" in the Sea of Cortez. Eastern Pacific Coral Reefs 26: 265-270.

Mostafavi PG, Fatemi SMR, Shahhosseiny MH, Hoegh-Guldberg O, Loh WKW (2007) Predominance of clade D Symbiodinium in shallow-water reef-building corals off Kish and Larak Islands (Persian Gulf, Iran). Mar Biol 153: 25-34.

Muscatine L, Porter JW (1977) Reef corals: mutualistic symbioses adapted to nutrient-poor environments. BioScience 27: 454-460.

Oliver TA, Palumbi SR (2011) Many corals host thermally resistant symbionts in high-temperature habitat. Coral Reefs 30: 241-250.

Panithanarak T (2012) Habitat partitioning of genetically distinct zooxanthellae inhabiting soft corals in the genus Sinularia. Proceedings of the Burapha University International Conference 2012, Pattaya, Thailand, 9-11 July, 2012, pp. 512-523.

Panithanarak T, Siriwong S, Putchakarn S, Dheerakamporn S (2011) Morphological and genetic variations of soft coral, $\mathrm{Si}$ nularia May, 1898 (Octocorallia: Alcyonacea) along NangRong Beach, Jorake Island and Juang Islands, Amphur Satta- 
hip, Chonburi Province. Final Report supported by a budget for research promotion from the Thai Government to Burapha University during 2009-2010, 82 pp. (in Thai)

Pochon W, Gates RD (2010) A new Symbiodinium clade (Dinophyceae) from soritid foraminifera in Hawaii. Mol Phylogenet Evol 56: 492-497.

Pochon X, Montoya-Burgos JI, Stadelmann B, Pawlowski J (2006) Molecular phylogeny, evolutionary rates, and divergence timing of the symbiotic dinoflagellate genus Symbiodinium. Mol Phylogenet Evol 38: 20-30.

Pochon X, Putnam HM, Burki F, Gates RD (2012) Identifying and characterizing alternative molecular markers for the symbiotic and free-living dinoflagellate genus Symbiodinium. PLoS ONE 7: e29816. doi: 10.1371/journal.pone.0029816.

Rowan R (2004) Coral bleaching-Thermal adaptation in reef coral symbionts. Nature 430: 742.

Saitou N, Nei M (1987) The neighbor-joining method: a new method for reconstructing phylogenetic trees. Mol Biol Evol 4: 406-425.

Sampayo EM, Ridgway T, Bongaerts P, Hoegh-Guldberg O (2008) Bleaching susceptibility and mortality of corals are determined by fine-scale differences in symbiont type. P Natl Acad Sci USA 105: 10444-10449.

Santos SR, Taylor DJ, Kinzie RA III, Hidaka M, Sakai K, Coffroth MA (2002) Molecular phylogeny of symbiotic dinoflagellates inferred from partial chloroplast large subunit (23S)rDNA sequences. Mol Phylogenet Evol 23: 97-111.

Stat M, Pochon X, Cowie ROM, Gates RD (2009) Specificity in communities of Symbiodinium in corals from Johnston Atoll. Mar Ecol Prog Ser 386: 83-96.

Swofford DL (2002) PAUP*-phylogenetic analysis using parsimony (*and other methods), Version 4.0b10. Sinauer Associ- ates, Sunderland, MA.

Thompson JD, Gibson TJ, Plewniak F, Jeanmougin F, Higgins DG (1997) The Clustal X windows interface: flexible strategies for multiple sequence alignment aided by quality analysis tools. Nucleic Acids Res 24: 4876-4882.

Tun K, Chou LM, Low J, Yeemin T, Phongsuwan N, Setiasih N, Wilson J, Amri AY, Adzis KAA, Lane D, van Bochove JW, Kluskens B, van Long N, Tuan VS, Gomez E (2010) A regional overview on the 2010 coral bleaching event in Southeast Asia. In: Status of Coral Reefs in East Asian Seas Region: 2010 (eds Kimura T, Tun K). Ministry of the Environment, Japan, pp. 16-27.

Van Oppen MJH, Mahini AJ, Done TJ (2005) Geographic distribution of zooxanthella types in three coral species on the Great Barrier Reef sampled after the 2002 bleaching event. Coral Reefs 24: 482-487.

Yeemin T, Mantachitra V, Plathong S, Nuclear P, Klinthong W, Sutthacheep M (2012) Impacts of coral bleaching, recovery and management in Thailand. Proceedings of the $12^{\text {th }}$ International Coral Reef Symposium, Cairns, Australia, 9-13 July 2012, p. 5.

Yeemin T, Saenghaisuk C, Sittiporn P, Klinthong W, Sangmanee K, Yucharoen M, Donsomjit W, Saengsawang L, Nuclear P, Sutthacheep M (2010) Status of coral reefs in Thailand following the 2010 coral bleaching event. In: Status of Coral Reefs in East Asian Seas Region: 2010 (eds Kimura T, Tun K). Ministry of the Environment, Japan, pp. 29-49.

Zhang Z, Green BR, Cavalier-Smith T (2000) Phylogeny of ultrarapidly evolving dinoflagellate chloroplast genes: a possible common origin for sporozoan and dinoflagellate plastids. J Mol Evol 51: 26-40. 